\title{
Quantitative measurement of telomerase reverse transcriptase mRNA and chromosomal analysis of urine by M-FISH in the diagnosis and follow-up of bladder cancer
}

\author{
THORAYA BADAWY ${ }^{1}$, SHAWKI EL-ABD ${ }^{2}$, MOHAMED ZAHRA $^{1}$, MANAL EID $^{1}$, \\ SAID ABDOU ${ }^{1}$ and SHERIEN EL-SHAZLY ${ }^{1}$ \\ Departments of ${ }^{1}$ Clinical Pathology and ${ }^{2}$ Urology, Tanta University, Tanta, Egypt
}

Received December 10, 2007; Accepted February 19, 2008

\begin{abstract}
This study was performed to evaluate the clinical utility of the measurement of the expression of telomerase enzyme (the catalytic subunit of the complex hTERT) and of the chromosomal analysis of urine by multi-color fluorescence in situ hybridization (M-FISH) assay for the detection of bladder cancer and its recurrence. These results were compared with those afforded by urine cytology, hematuria screening and the bladder tumor antigen (BTA) and fibrin degradation products (FDP) tests. Urine samples were obtained from three groups: 30 patients with bladder cancer, 15 patients with nonmalignant bladder disorders and 8 healthy individuals. hTERT mRNA was measured by reverse transcription real-time PCR. M-FISH was performed using a mixture of fluorescent labeled probes for the centromeric regions of chromosomes $3,7,17$, and the locus specific identifier p16 probe for the 9p21 locus. We demonstrated that the overall sensitivity of hemoglobin dipstick and the BTA and FDP tests was insignificantly greater than that of urine cytology, but with a lower specificity. The hTERT mRNA expression marker offered significantly greater sensitivity for detecting all bladder cancers, especially superficial and low grade tumors, than did urine cytology, and with a higher specificity. M-FISH was significantly more sensitive than urine cytology in detecting bladder tumors, but had lower specificity (insignificant results). The superior sensi-
\end{abstract}

Correspondence to: Dr Manal Abd-Elwahed Eid, Department of Clinical Pathology, Tanta University, Tanta, Egypt

E-mail: manaleid@hotmail.com

Abbreviations: BTA, bladder tumor antigen; CEP, chromosome enumeration probe; DAPI, 4',6-diamidino-2-phenylindol; EGFR, epidermal growth factor receptor; FDP, fibrin degradation products; GAPDH, glyceraldehyde-3-phosphate-dehydrogenase; hTERT, human telomerase reverse transcriptase unit; M-FISH, multi-color fluorescence in situ hybridization; SCC, squamous cell carcinoma; TCC, transitional cell carcinoma

Key words: telomerase reverse transcriptase, fluorescence in situ hybridization, bladder cancer tivity of M-FISH was maintained when comparing the two assays in terms of low-stage and low grade tumor detection. M-FISH was the most sensitive and specific test in detecting tumor recurrence, followed by the BTA test. hTERT, hematuria screening and FDP showed relatively low sensitivity and low specificity during follow-up. Our findings suggest that the assessment of hTERT expression and chromosomal abnormalities in urine represent reliable tools - equally specific yet far more sensitive than conventional cytology - for the early detection of bladder cancer. The high sensitivity of FISH in detecting recurrence makes it useful for reducing the number of cyctoscopies usually performed in the accurate follow-up of these cases.

\section{Introduction}

Bladder cancer is one of the most prominent cancers in the world, and one of the most prevalent cancers in Egypt. The National Cancer Institute (NCI), Cairo, reported that bladder cancer constitutes $30.3 \%$ of all cancers in Egypt $(1,2)$. As Egypt represents a hyperendemic area for schistosome infection, schistosome-related bladder cancer accounts for $82.4 \%$ of all bladder cancers $(3,4)$. Almost $30-50 \%$ of newly-diagnosed patients have superficial tumors limited to the mucosa or submucosa. Such cases, when the disease is detected before it invades the bladder muscle, usually have a good prognosis. Unfortunately, the recurrence rate of bladder cancer is $50-70 \%$, and $20-30 \%$ of recurrent tumors progress to muscle invasive disease. Consequently, patients must receive regular follow-up exams at 3-6 month intervals (5).

The current methods of evaluation for initial diagnosis and follow-up involve cystoscopy and biopsy, an invasive procedure. It may also be inconclusive at times, especially in the numerous patients with an indwelling catheter or active inflammation, where it may not be definitive due to the grossly abnormal appearance of the bladder mucosa. Cytoscopy is therefore not ideal for the screening and follow-up of bladder cancer; what is needed is a non-invasive method with high sensitivity, specificity and predictive values for bladder cancer detection, and for the identification of patients at high risk of tumor recurrence or progression $(6,7)$.

There are several situations during diagnosis and the selection of appropriate treatment for bladder cancer where 
objective markers could potentially transform clinical practice. Several attempts have been made to improve the detection of cancer cells in urine samples by multiple urinary tumor markers, such as nuclear matrix protein (NMP)22, DNA ploidy and cytokeratins; however, the sensitivity of these markers is too low to be introduced to clinical practice, indicating the need for new markers (8). Molecular markers are now a source of great hope. Identification of the genetic and chromosomal changes that are fundamental to the malignant process in bladder cancer would be useful for developing detection and preventive strategies, while identifying those changes that are secondary to the malignant process may be more valuable for prognostic purposes. Awareness of both is likely needed in order to develop effective therapeutic approaches (9).

Bladder cancer initiation and development involves genetic changes and chromosomal aberrations that often lead to either the deletion of tumor suppressor genes or the induction of oncogenes. This increases the proliferative potential of tumor cells and their resistance to apoptosis. However, these processes alone do not ensure tumor growth. After successive replication, cells lose their protective telomere ends, resulting in chromosomal shortening and cell death. Malignant cells are able to maintain telomere length above a critical threshold by up-regulating the expression of the enzyme telomerase, which catalyzes the addition of telomeric repeats to the 3 '-end of the chromosome to allow unlimited replication potential (9). Telomerase, a ribonucleoprotein enzyme, needs at least two components to be functional: the RNA component, which is coded by the human telomerase RNA $(h T R)$ gene and serves as a template for telomere DNA synthesis, and the human telomerase reverse transcriptase subunit ( $h T E R T$ gene), which codes for the catalytic subunit of the enzyme. Telomerase activity is the most general molecular marker for the identification of human cancer and can be detected in $85 \%$ of all tumors, whereas little or no telomerase expression is found in healthy tissues. A method for the quantitative measurement of hTERT mRNA expression may be of import to molecular diagnosis in tumors and corresponding bodily samples (10).

Bladder tumor initiation and progression is accompanied by increased chromosomal instability and aneuploidy. Cytogenetic studies on bladder cancer have revealed frequent alterations of a variety of chromosomes, including chromosomes 9, 17, 7, 11, 1,8 and others. Fluorescence in situ hybridization (FISH) can be used to detect cells with these chromosomal alterations (11).

In the present study, we quantified telomerase reverse transcriptase mRNA and screened for the presence of chromosomal aberration involving chromosomes 3, 7, 17, and 9 in the urine of bladder cancer patients and those at risk of developing bladder cancer. This was in order to shed light on their clinicopathological significance in the diagnosis and follow-up of the disease, and examine their relation to other routine diagnostic markers that use a non-invasive route.

\section{Patients and methods}

Subject selection. The study was conducted on 45 patients, with 8 apparently healthy individuals serving as the control group. All patients were selected from the Urology department of Tanta University, and consent was obtained from all subjects included in the study. The 45 patients included 15 with nonneoplastic bladder disorders (6 of whom were diagnosed with
Bilharzial cystitis, 3 with non-Bilharzial chronic cystitis and 6 with bladder stones and cystitis). Of these, 11 were male and 4 female, with ages ranging 40-58 years (mean 52.8 \pm 5.1 ). The other 30 patients were newly diagnosed with bladder cancer and included 21 males and 9 females, with ages ranging

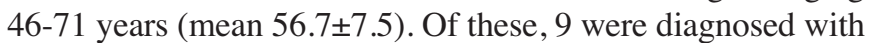
Bilharzial squamous cell carcinoma (SCC), 7 with Bilharzial transitional cell carcinoma (TCC) and 14 with non-Bilharzial TCC. Patients were divided according to disease stage: 13 cases of superficial carcinoma ( 3 pTa and 10 pT1) and 17 of invasive tumors (pT2-pT4). In terms of histopathological grade, 6 patients were grade I, 13 were grade II and 11 were grade III. None of the patients had a history of upper urinary tract disorders, as confirmed by radiological and laboratory examinations. The diagnosis of bladder cancer was based on clinical, radiological, cystoscopy and histological examinations. Those cases associated with cyctitis were first subjected to urine culture and sensitivity tests for proper antibiotic treatment before the initiation of the study. The control group included 6 males and 2 females, with ages ranging 40-63 years (mean 51 \pm 8.2 ).

All subjects involved in the study were subjected to routine examinations, including clinical examination, radiological studies, urine analysis, kidney function tests and the indirect heamagglutination test for Schistosomiasis. They also underwent specific examinations, including cyctoscopy and biopsy for urinary bladder masses, urine cytological examination, hematuria screening by hemoglobin dipsticks (Hemastix, Bayer Diagnostic, USA), detection of bladder tumor antigen in urine using the bladder tumor antigen (BTA) stat test (Polymedco Inc., USA), detection of fibrin degradation products (FDP) in urine using the latex semi-quantitative thrombo-Wellcotest kit (Murex, USA), a quantitative assay of telomerase reverse transcriptase mRNA (hTERT mRNA) in urine using real-time RT-PCR and screening for aberrations, including chromosomes 3, 7, 17 and the 9p21 locus in urine samples, by UroVysion multi-color fluorescence in situ hybridization (M-FISH).

Specimen collection. Morning urine samples were collected for cytological examination. About $100 \mathrm{ml}$ of the second morning samples were collected into autoclaved collection tubes for routine and specific investigations. Each urine sample was divided as follows: $10 \mathrm{ml}$ for routine urine analysis, $5 \mathrm{ml}$ for culture and sensitivity if indicated, $2 \mathrm{ml}$ for hemoglobin dipstick, $2 \mathrm{ml}$ for FDP detection, a few drops for the BTA stat test, $25 \mathrm{ml}$ for RNA extraction and subsequent hTERT mRNA quantitation, and $50 \mathrm{ml}$ for FISH.

Patient follow-up. Follow-up was done at one month and then every three months for one year following management. For the first two visits, follow-up consisted of cystoscopy, urine cytology, hemoglobin dipstick, the FDP test, the BTA stat test, quantitative assay of hTERT mRNA, and chromosomal assay. Subsequently, follow-up was done by cystoscopy every three months when possible.

Quantitation of hTERT mRNA. Quantitation of hTERT gene expression was performed by two-step quantitative real-time RT-PCR. In the reverse transcription step, cDNA was reverse transcribed from extracted RNA using random hexamers. In 
the real-time polymerase chain reaction step, products were amplified from cDNA using the AmpliTaq Gold DNA polymerase, TaqMan PDARs primers and TaqMan fluorescent probe (Applied Biosystems, USA).

Total-RNA extraction. Total-RNA was extracted from cell pellets harvested from the urine samples using a combination of TRIzol reagent and the Qiagen RNeasy mini spin column (RNeasy Mini Kit, Qiagen, USA) as described previously. Briefly, TRIzol and chloroform were added to the cell pellet, which was then incubated for $15 \mathrm{~min}$ and centrifuged. The upper aqueous phase was transferred to the RNeasy mini spin column. The columns were washed and RNA was eluted in RNase-free water $(12,13)$.

Real-time quantitative $R T-P C R$. For the quantitation of hTERT mRNA using real-time RT-PCR, a calibration curve of human reference total-RNA (Applied Biosystems) was used. Reference total-RNA is composed of total-RNA from 10 human cell lines, with quantities of RNA from each cell line optimized for maximum representation of hTERT mRNA. In order to normalize sample-to-sample differences in RNA input, RNA quality and RT efficiency, the level of glyceraldehyde-3-phosphate-dehydrogenase (GAPDH) expression was quantitatively measured in each sample with a GAPDH standard curve.

First-step PCR (cDNA synthesis). cDNA was reverse transcripted from the total-RNA of the urine samples and from the stock RNA of the human reference total-RNA using random hexamers (High-Capacity cDNA kit, Applied Biosystems) under the following conditions: hexanucleotides annealing for $10 \mathrm{~min}$ at $25^{\circ} \mathrm{C}$, cDNA synthesis for $30 \mathrm{~min}$ at $48^{\circ} \mathrm{C}$, then enzyme inactivation for $5 \mathrm{~min}$ at $95^{\circ} \mathrm{C}$. The cDNA of the subjects was stored at $-70^{\circ} \mathrm{C}$ until use. Two sets of 2 -fold dilution series (50-3.125 ng/ $\mu \mathrm{l}$ ) of human reference cDNA were prepared in RNase-free $\mathrm{H}_{2} \mathrm{O}$ and stored at $-70^{\circ} \mathrm{C}$ for later use (14).

Second-step PCR (cDNA amplification). AmpliTaq Gold DNA polymerase was used to amplify cDNA using TaqMan primers, TaqMan fluorescent probes and TaqMan universal PCR master mix (Applied Biosystems). The sequences of the primers and of the hTERT probe were: forward primer, 5'CGGAAGAGTGTCTGGAGCAA-3'; reverse primer, 5'-GGA TGAAGCGGAGTCTGGA-3'; probe: Fam-TGCAAAGCA TTGGAATCAGACAGCACT-Quencher MGB. Amplification of GAPDH was performed for each sample in a separate tube using the following primers and probe: forward primer, 5'GAAGGTGAAGGTC GGAGTC-3'; reverse primer, 5'-GAA GATGGTGATGGGATTTC-3'; probe: VIC-CAAGCTTCC CGTTCTCAGCC-TAMRA.

The TaqMan universal PCR master mix contains AmpliTaq Gold DNA polymerase, AmpErase UNG, dNTPs with dUTP, and optimized buffer. The reaction mixture was performed according to the manufacturer's instructions in a final volume of $50 \mu 1$ under the following conditions: initial UNG incubation at $50^{\circ} \mathrm{C}$ for $2 \mathrm{~min}$, enzyme activation at $95^{\circ} \mathrm{C}$ for $10 \mathrm{~min}$, 40 cycles of denaturation at $95^{\circ} \mathrm{C}$ for $15 \mathrm{sec}$, and annealing/ extension at $60^{\circ} \mathrm{C}$ for $1 \mathrm{~min}$. The assay was performed utilizing the GeneAmp 5700 Sequence Detection System (Applied Biosystems). The amounts of hTERT and GAPDH mRNA were determined from the corresponding standard curves and the hTERT mRNA was divided by the corresponding GAPDH reference amount to obtain a normalized hTERT mRNA value (15).

Detection of aberration in chromosomes 3, 7, 17 and the 9p21 locus. M-FISH (UroVysion Kit, Vysis, USA) was designed to detect aneuploidy in chromosomes 3, 7 and 17, and the loss of the 9p21 locus in urine. The mixture consists of four labeled probes. These include three $\alpha$-satellite repeat sequence probes: chromosome enumeration probe (CEP) 3 spectrum red, CEP7 spectrum green and CEP17 spectrum aqua, which hybridize to the centromer regions of chromosomes 3, 7 and 17 respectively, and a unique sequence probe: locus specific identifier p16 spectrum gold, which hybridizes to the p16 gene at the 9 p21 locus. Cell pellets from fresh urine samples were fixed on slides. DNA was denatured to its single-strand form and allowed to hybridize with the UroVysion probes. Following hybridization, the unbound probes were removed by a series of washes and the nuclei were counterstained with 4',6-diamidino2-phenylindol (DAPI) fluorescent stain. The probes were then viewed on an Olympus fluorescence microscope BX60, equipped with a filter set including Aqua single bandpass (chromosome 17), Gold single bandpass (the 9p21 locus) and Red/Green/DAPI triple bandpass (chromosomes 3 and 7) $(11,16)$.

The slides were scored for hybridization signals according to the manufacturer's protocol. Enumeration of FISH signals was done on target cells that appeared morphologically abnormal (large nuclear size, irregular nuclear shape and patchy DAPI staining). Cells were defined as FISH-positive if they showed three or more signals of two or more of the Aqua (CEP 17), Green (CEP 7) or Red (CEP 3) probes and/or a loss of both gold signals (9p21). For each specimen, a minimum of 25 cells was analyzed. If fewer than 25 target cells were found, the remaining cells were marked as disomic (i.e., 2-2-2-2). Control slides were run concurrently with patient slides to monitor assay performance and to assess the accuracy of signal viewing (UroVysion control slides, Vysis).

The cut-off for FISH-positive bladder cancer specimens was performed according to the criteria suggested by Mayo's Cytogenetics Laboratory, 2002. Accordingly, a specimen was considered FISH-positive for bladder cancer if at least one of the following criteria was met: i) $\geq 5$ of the 25 examined cells exhibiting a gain of 2 or more chromosomes (3, 7 or 17) in the same cell, or ii) $\geq 12$ of the 25 cells exhibiting zero 9 p21 signals (both copies lost) (17).

\section{Results}

Utility of urine cytology, hematuria, BTA and FDP screening in the detection of bladder cancer. Forty-five patients (15 with benign bladder disorders and 30 with bladder cancer) and 8 healthy individuals were included in the study. Their urine samples were subjected to cytological examination, the measurement of BTA, hematuria and FDP (Tables I and II).

Cytological examination was negative for malignancy in all control subjects and in $13(86.6 \%)$ of the 15 benign cases. 
Table I. Significance of urine markers in the diagnosis of bladder cancer.

\begin{tabular}{lcccccc}
\hline & Cytology & Hb Dipstick & BTA stat & FDP & hTERT mRNA & M-FISH \\
\hline Sensitivity (\%) & 53.3 & 76.7 & 70.0 & 70.0 & 93.3 & 90.0 \\
Specificity (\%) & 92.5 & 14.3 & 64.3 & 71.4 & 100.0 & 85.7 \\
PPV (\%) & 94.1 & 65.7 & 80.7 & 84.0 & 100.0 & 93.1 \\
NPV (\%) & 48.1 & 22.2 & 50.0 & 52.6 & 87.5 & 80.0 \\
Accuracy (\%) & 85.3 & 56.8 & 68.2 & 70.4 & 95.5 & 88.6 \\
\hline
\end{tabular}

PPV, positive predictive value; NPV, negative predictive value.

Table II. Sensitivity of the different urine markers studied to various histopathological features of bladder cancer.

\begin{tabular}{|c|c|c|c|c|c|c|}
\hline $\begin{array}{l}\text { Histopathological } \\
\text { features }\end{array}$ & $\begin{array}{l}\text { Cytology } \\
(\%)\end{array}$ & $\begin{array}{c}\text { Hb Dipstick } \\
(\%)\end{array}$ & $\begin{array}{l}\text { BTA stat } \\
(\%)\end{array}$ & $\begin{array}{l}\text { FDP } \\
(\%)\end{array}$ & $\begin{array}{l}\text { hTERT } \\
(\%)\end{array}$ & $\begin{array}{c}\text { M-FISH } \\
(\%)\end{array}$ \\
\hline \multicolumn{7}{|l|}{ Pathological stage } \\
\hline \multicolumn{7}{|l|}{ Superficial tumors } \\
\hline $\mathrm{pTa}(\mathrm{n}=3)$ & 0.0 & 33.3 & 33.3 & 33.3 & 66.7 & 33.3 \\
\hline pT1 $(n=10)$ & 50.0 & 60.0 & 50.0 & 60.0 & 90.0 & 90.0 \\
\hline \multicolumn{7}{|l|}{ Invasive tumors } \\
\hline$(\mathrm{pT} 2-4)(\mathrm{n}=17)$ & 64.7 & 94.1 & 88.2 & 82.3 & 100.0 & 100.0 \\
\hline \multicolumn{7}{|l|}{ Pathological grade } \\
\hline$I(n=6)$ & 16.7 & 33.3 & 16.7 & 33.3 & 83.3 & 50.0 \\
\hline II $(n=13)$ & 53.8 & 84.6 & 69.2 & 69.2 & 92.3 & 100.0 \\
\hline III $(n=11)$ & 72.7 & 90.9 & 100.0 & 90.9 & 100.0 & 100.0 \\
\hline \multicolumn{7}{|l|}{ Pathological type } \\
\hline Bilharzial SCC (n=9) & 44.4 & 77.8 & 55.6 & 77.8 & 100.0 & 100.0 \\
\hline Bilharzial TCC (n=7) & 57.1 & 85.7 & 71.4 & 71.4 & 85.7 & 85.7 \\
\hline Non-Bilharzial TCC (n=14) & 57.1 & 85.7 & 78.6 & 64.3 & 92.9 & 85.7 \\
\hline
\end{tabular}

PPV, positive predictive value; NPV, negative predictive value.

Two benign cases were suspected of having malignant cells by urine cytology. This was disproved by biopsy and histopathological examination. Urine cytology was positive for malignancy in 16 (sensitivity $53.3 \%$ ) of the 30 patients with bladder cancer examined. It afforded an accuracy of $85.3 \%$, a specificity of $92.5 \%$, a positive predictive value (PPV) of $94.1 \%$ and a negative predictive value (NPV) of $48.1 \%$. Urine cytology was markedly insensitive in detecting superficial tumors (a sensitivity of 0 and $50 \%$ for tumor stages pTa and pT1, respectively). Its sensitivity increased to $64.7 \%$ when detecting invasive tumors $(\mathrm{P}>0.05)$. Similarly, the sensitivity of urine cytology was very low when detecting grade I tumors (16.7\%), but increased to 53.8 and $72.7 \%$ for tumor grades II and III, respectively $(\mathrm{P}>0.05)$. No significant difference was observed in the sensitivity of urine cytology to the different pathological tumor types (44.4\% for Bilharzial SCC and 57.1\% for both Bilharzial and non-Bilharzial TCC, P>0.05).

Hemoglobin was measured in urine by semi-quantitative Hemastix assay. None of the control subjects were positive for urine hemoglobin. The test was positive in 12/14 (85.7\%) of the patients with benign disorders and in 23/30 (76.6\%) of the bladder cancer cases. Despite the considerable sensitivity of the test in detecting bladder cancer $(76.6 \%)$, it showed very poor specificity (14.3\%), accuracy $(56.8 \%)$, PPV (65.7\%) and NPV (22.2\%). As was the case with urine cytology, urine hemoglobin was significantly insensitive in detecting superficial tumors (sensitivity 33.3, 60 and $94.1 \%$ for pTa, pT1 and invasive tumors, respectively; $\mathrm{P}<0.05)$. Similarly, the test was significantly more sensitive to higher tumor grades than lower ones [sensitivity $33.3(\mathrm{P}<0.05), 84.6$ and $90.9 \%(\mathrm{P}>0.05)$ for tumor grades I, II and III, respectively]. No difference was observed in the sensitivity of the hemoglobin test to the different histological tumor types (sensitivity $77.8 \%$ for Bilharzial SCC and $85.7 \%$ for Bilharzial and non-Bilharzial TCC; $\mathrm{P}>0.05)$.

All control subjects were negative for urine BTA. The antigen was detected in the urine of $5(35.7 \%)$ benign cases and $21(70 \%)$ bladder cancer cases. The specificity of the test for 
the diagnosis of bladder cancer was $64.3 \%$, the PPV $80.7 \%$, the NPV $50 \%$ and the accuracy $68.2 \%$. The test had a significantly higher sensitivity in detecting invasive tumors compared to superficial ones, and in detecting high grade tumors compared to low grade ones [sensitivity 33.3, 50 and $88.2 \%$ for tumor stages pTa, pT1 and pT2-4, respectively $(\mathrm{P}<0.05)$, and 16.7, 69.2 and $100 \%$ for tumor grades I, II and III, respectively $(\mathrm{P}<0.05)]$. No significant difference in BTA sensitivity to different histological types (55.6, 71.4 and 78.6\% for Bilharzial SCC, Bilharzial TCC and non-Bilharzial TCC, respectively; $\mathrm{P}>0.05$ ) was observed.

Similar to the previous assays, urine FDP was positive in $4(26.5 \%)$ benign cases and $21(70 \%)$ bladder cancer cases. It had a specificity of $71.4 \%$, a PPV of $84 \%$, an NPV of $52.6 \%$ and an accuracy of $70.4 \%$ in detecting bladder cancer. It showed lower sensitivity when detecting low grade and superficial tumors, but no difference when detecting different pathological types [sensitivity 33.3, 60 and $82.3 \%$ for pTa, pT1 and pT2-4, respectively ( $\mathrm{P}>0.05), 33.3,69.2$ and $90.9 \%$ for grades I, II and III, respectively (P>0.05), and 77.8, 71.4 and $64.3 \%$ for Bilharzial SCC, Bilharzial TCC and non-Bilharzial TCC, respectively $(\mathrm{P}>0.05)]$.

Expression of hTERT mRNA in bladder cancer. hTERT mRNA was measured in urine sedimented cells using quantitative real-time PCR and normalized to GAPDH expression. None of the healthy individuals expressed hTERT. Four (26.6\%) of the 15 benign cases showed very low hTERT expression (range $0.03-0.57$; mean $0.12 \pm 0.1$ ) with no significant difference compared to the controls ( $\mathrm{P}>0.05)$. Three of the 4 benign cases expressing hTERT showed cytologic atypia. On the other hand, $28(93.3 \%)$ of the 30 bladder cancer cases demonstrated significantly high hTERT expression (range 0.33-13.58; mean $4.3 \pm 3.01 ; \mathrm{P}<0.05$ ), yielding an overall specificity of $100 \%$, a PPV of $100 \%$, an NPV of $87.5 \%$ and an accuracy of $95.5 \%$ in the diagnosis of bladder cancer. Notably, hTERT expression was as sensitive when detecting superficial and low grade tumors as when detecting invasive high grade ones [sensitivity 66.7, 90 and 100\% for tumors pTa, pT1 and pT2-4, respectively ( $\mathrm{P}>0.05)$, and 83.3, 92.3 and $100 \%$ for tumor grades I, II and III, respectively $(\mathrm{P}>0.05)]$. No significant difference was observed in the sensitivity of the test in detecting different tumor types (100, 85.7 and $92.9 \%$ for Bilharzial SCC, Bilharzial TCC and non-Bilharzial TCC, respectively; P>0.05). The estimated cut-off between non-neoplastic and neoplastic cases was $>0.57$, the cut-off between superficial and invasive tumors $\geq 3.7$, and that between low and high grades $>4.2$ (Fig. 1).

Detection of chromosomal aberration in urine sedimented cells. Cells collected from the second morning urine samples were fixed on slides and examined by M-FISH for the presence of chromosome 3, 9, 7 and 17 aberrations. The slides were considered FISH-positive for bladder cancer if a gain of $\geq 2$ chromosomes (3, 7 and/or 17) was present in the same cell, or if $\geq 12$ of the 25 examined cells showed homozygous deletion of 9p21 (Mayo Cytogenetics Laboratory protocol, 2002). All control subjects were free of chromosomal aberrations. Of the benign cases, $3(20 \%)$ demonstrated 9p21 deletion. Notably, they were the same cases which showed

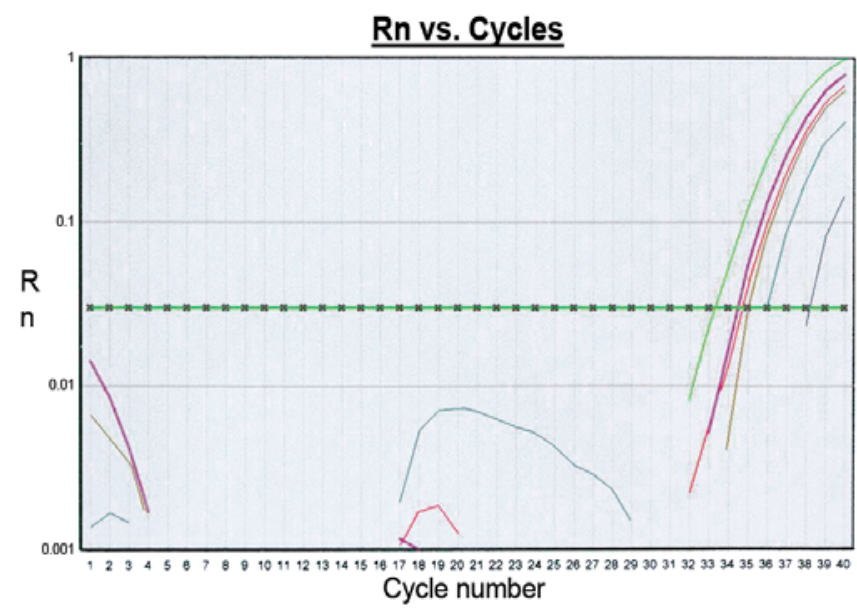

Figure 1. Real-time PCR amplification blot for hTERT mRNA in bladder cancer patients.

minimal expression of hTERT. Among the 30 cases of bladder cancer, 27 (90\%) demonstrated chromosomal abnormalities. The specificity of FISH for the detection of bladder cancer was $85.7 \%$, the PPV $93.1 \%$, the NPV $80 \%$ and the accuracy $88.6 \%$. FISH was significantly less sensitive when detecting superficial and low grade tumors [sensitivity 33.3, 90 and $100 \%$ for $\mathrm{pTa}$, pT1 and pT2-4, respectively $(\mathrm{P}<0.05)$ for pT2-4 and pT1 compared to pTa, and 50, 100 and $100 \%$ for tumor grades I, II and III, respectively $(\mathrm{P}<0.05)$ for grades II and III compared to grade I]. No significant difference in sensitivity to different pathological tumor types $(100,85.7$ and $85.7 \%$ for Bilharzial SCC, Bilharzial TCC and non-Bilharzial TCC, respectively) was observed.

Utility of urine markers in the detection and prediction of bladder cancer recurrence. As previously mentioned, followup was performed for 12 months. Only 20 patients could be followed after the start of therapy. In 10 of these, recurrence with a higher stage or grade tumor was detected within 4-12 months.

In terms of cytological examination and hematuria screening, 12 cases presented as positive during follow-up. Only 4 of these were histopathologically confirmed as tumor recurrence. Of the 8 urine cytology and hematuria-negative patients, 6 developed recurrence. The FDP test recorded positive results in 10 of the 20 follow-up patients, only 4 of which were histopathologically proven to be true positive. Six of the 10 FDP-negative patients demonstrated tumor recurrence. During follow-up, hTERT was detected in 8 patients, of which only 4 were confirmed as true positive. Of the 12 hTERT-negative patients followed, 6 developed recurrence. Despite a lack of significance, the BTA test was more sensitive and more specific in detecting recurrence than the other tests. It recorded positive results in 12 of the 20 follow-up patients, 8 of which were histopathologically confirmed as true positive. Of the 8 BTA-negative patients, only 2 developed recurrence. Detection of chromosomal aberration using M-FISH (Fig. 2) was the most sensitive and specific test for detecting tumor recurrence as compared to the other urine markers studied, exhibiting statistically significant utility for the prediction of 

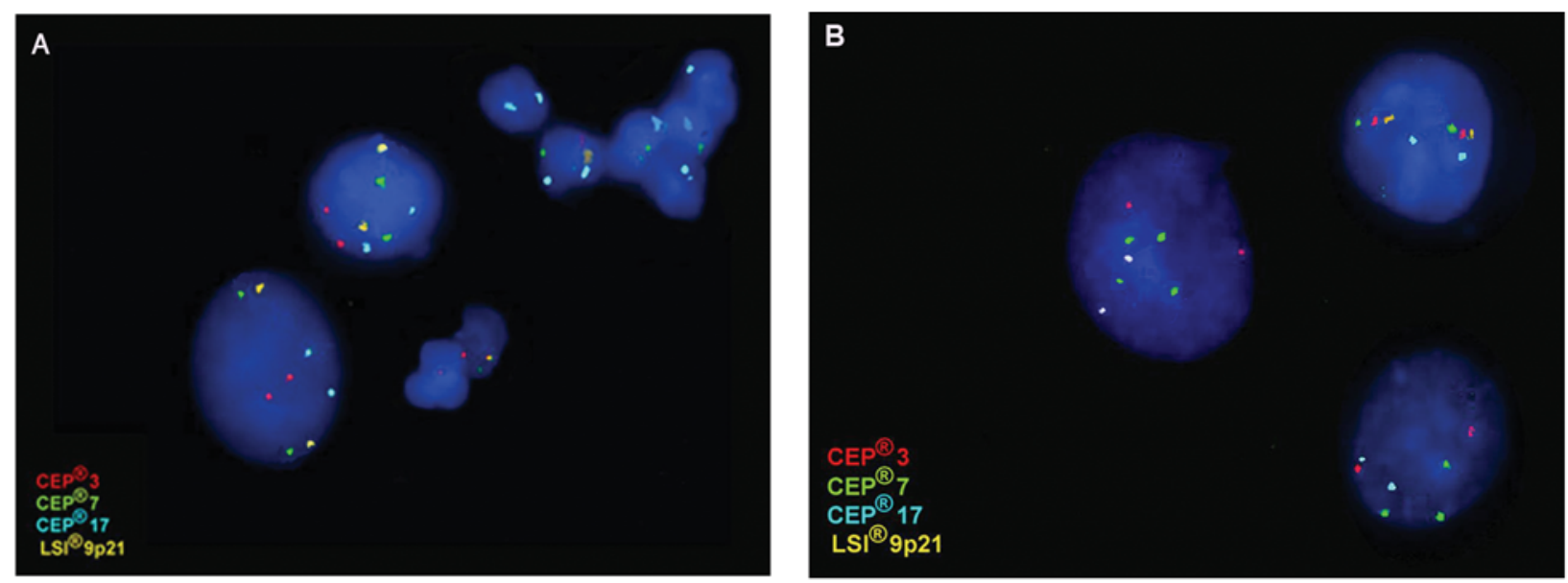

Figure 2. M-FISH. (A) A chronic cystitis case with a duplicate number for all signals. (B) A bladder cancer case. Cells with polysomy 7 are marked in green and homozygous deletion of $9 \mathrm{p} 21$ is in yellow.

Table III. Significance of urine markers in the detection of bladder cancer recurrence.

\begin{tabular}{lcccccc}
\hline & Cytology & Hb Dipstick & BTA stat & FDP & Normalized hTERT & M-FISH \\
\hline True positive & 4 & 4 & 8 & 4 & 4 & 10 \\
False positive & 8 & 8 & 4 & 6 & 4 & 2 \\
P-value & $>0.05$ & $>0.05$ & $>0.05$ & $>0.05$ & $>0.05$ & $>0.05$ \\
True negative & 2 & 2 & 6 & 4 & 6 & 8 \\
False negative & 6 & 6 & 2 & 6 & 6 & 0 \\
P-value & $>0.05$ & $>0.05$ & $>0.05$ & $>0.05$ & $>0.05$ & $<0.05$ \\
Sensitivity (\%) & 40 & 40 & 80 & 40 & 40 & 100 \\
Specificity (\%) & 20 & 20 & 60 & 40 & 60 & 80 \\
PPV (\%) & 33.3 & 33.3 & 66.7 & 40 & 50 & 83.3 \\
NPV (\%) & 25 & 25 & 75 & 40 & 50 & 100 \\
Accuracy (\%) & 30 & 30 & 70 & 40 & 50 & 90 \\
\hline
\end{tabular}

PPV, positive predictive value; NPV, negative predictive value.

recurrence. Of the 20 follow-up patients, 12 recorded as FISHpositive. Ten of these were histopathologically confirmed. None of the 8 FISH-negative patients developed recurrence. The significance of the urine markers studied for the detection of bladder cancer recurrence is summarized in Table III.

Statistical analysis. Analysis of the data was performed using SPSS version 11 software. The unpaired Student's t-test was used to test the significance between the quantitative data of two groups. ANOVA was used to compare quantitative data among different groups. The list significant difference test was used to compare quantitative data between two groups if the ANOVA was significant. The comparison of non-parametric quantitative data in two different groups using their mean rank was performed using the Mann-Whitney test. The $\chi^{2}$ test was used for the comparison of qualitative variables in two or more groups. Receiver-operating characteristic curves were used to calculate the specificity, sensitivity and accuracy of cut-off value between two groups (18).

\section{Discussion}

At present, the standard procedure for the early detection of bladder cancer includes cystoscopy and urine cytology. The high rate of recurrence of these tumors, together with the frequent equivocal results obtained by urine cytology and the limited utility of cytology for detecting low stage and grade bladder cancer, implies that regular invasive cystoscopy is still required to diagnose and monitor patients for recurrence and progression. Non-invasive diagnostic tools that overcome these limitations would be of great import (19). Various alternative laboratory tests based on the detection of different substances, such as BTA, FDP and hyaluronic acid, have become available for the diagnosis of bladder cancer, in an attempt to improve on the diagnostic accuracy of cytology. However, the low in comparison with urine cytology sensitivity and unsatisfactory specificity reported have limited their clinical utility; none have proven capable of replacing urine cytology in the management of bladder cancer (20). Recent 
studies on urine markers reported that the evaluation of hTERT expression and chromosomal aberrations would be more useful for early bladder cancer detection $(21,22)$. In the present study, the performance of non-invasive quantitative measurement of hTERT expression and of the FISH assay in urine for the detection of different stages and grades of bladder cancer was evaluated in schistosome-related and non-schistosome-related bladder cancers, and compared to the performance of conventional urine cytology and other urine markers.

Bilharzia-associated bladder cancer represented $53.3 \%$ of all the cases studied, a finding which was expected due to the high prevalence of Bilharzial infection (a risk factor for bladder cancer) among Egyptians as reported in many studies (23). However, unlike most previous studies, TCC represented $70 \%$ of the bladder cancers studied, and the prevalence of Bilharzial TCC (43.2\%) was almost equal to that of Bilharzial SCC $(56.8 \%)$. In most other studies in Egypt, SCC was the more common type of bladder cancer, representing about two thirds of Bilharzial cases $(23,24)$. Supporting our results, NCI, Cairo has reported that the pattern of bladder cancer in Egypt began changing in 1993, and that by 2005 TCC represented $62.8 \%$ of all recorded cases. The rising incidence of TCC in Bilharzia-associated bladder cancer could be due to appearance of new environmental risk factors among Egyptians. This calls for further study (25).

The sensitivities obtained by cytology in the present study were quite similar to the sensitivity ranges reported in the literature $(11,26)$, with good sensitivity in detecting high stage and grade tumors, but insufficient sensitivity in the case of superficial and well-differentiated tumors. This could be attributed to the fact that it is very hard to distinguish welldifferentiated tumors from reactive urothelial changes in urine samples (6). In urine cytology, hematuria screening was more sensitive to high stage and grade tumors, but showed very poor specificity for superficial and low grade tumors. Similar results were reported by Halling et al (11). Hematuria has low specificity because it is a common finding in both malignant and non-malignant genitourinary disorders, such as stones and infections.

BTA, a human complement factor $\mathrm{H}$-related protein (hCFHrp), is produced by cancer cells. It interacts with complement factor $\mathrm{C} 3 \mathrm{~b}$ to inhibit the formation of the membrane attack complex, preventing cancer cell lysis and allowing the invasion of the host immune system (27). Although the BTA test showed better overall sensitivity than cytology for detecting bladder cancer, it had lower specificity with a high falsepositive rate. This could be related to hematuria, as all falsepositive BTA cases were associated with positive Hemastix tests. Similar observations were reported by Halling et al, who explained that the false-positive of BTA was associated with hematuria due to the fact that the plasma human complement factor $\mathrm{H}$ protein could be recognized by the antibodies of the BTA test (11). In the present study, the FDP test had similar overall sensitivity to BTA but a higher specificity, though still lower than that of cytology. Increased angiogenic factors in malignancy lead to an increase in vascular permeability and cause the leakage of plasma protein, including plasminogen, fibrinogen and other clotting factors, in extravascular tissue. Clotting factors convert fibrinogen into an extravascular fibrin clot, which is degraded by plasmin into FDP. However, various non-specific urinary inflammatory conditions can also result in detectable amounts of FDP in the urine (28). As is the case with most of urine markers, the sensitivity of BTA and FDP declined significantly with decreasing grade and stage, with no difference between pathological tumor types. Several previous studies support our findings $(11,29)$. In contrast, Khaled et al reported very high sensitivity (99\%) for BTA in detecting Bilharzia-associated bladder cancer (27). Moreover, Oeda and Manabe reported high sensitivity for the FDP test in detecting low grade $(70 \%)$ and non-invasive $(75 \%)$ tumors, and recommended FDP as a marker for bladder cancer screening. The use of the sensitive AuraTek immunoassay in their study may be responsible for this disagreement (30).

Studies on the activation and/or expression of human telomerase in urine have generated promising results regarding its use as a non-invasive surveillance tool for early bladder cancer detection. In the present study, hTERT expression was demonstrated in $93.3 \%$ of bladder cancers, and recorded the highest sensitivity for detecting superficial $(66.7 \%)$ and low grade $(83.3 \%)$ ones. It should be noted that similar or occasionally better rates have been reported by other study groups using similar and different methods, such as the telomeric repeat amplification protocol (TRAP) assay $(31,32)$. Moreover, Breslow et al reported that increased telomerase activity is associated with the acquisition of malignancy, and may have potential as a biomarker for the early detection of cancer (33).

Our study showed minimal hTERT expression in four benign cases; we therefore established a cut-off value $>0.57 \mathrm{ng} / \mu 1$ for bladder cancer diagnosis. One of these cases had suspicious cytology and another was M-FISH-positive for bladder cancer. The detection of hTERT expression in these benign lesions could be due to presence of cells with malignant potential, prior to development of morphologic changes. Alternatively, the lesions may have a small subset of malignant cells at the time of molecular investigation, or the lesions could be infiltrated by activated lymphocytes, which have been documented to show transient telomerase activity (31). Similar results were reported by BialkowskaHobrzanska et al (21). All their benign cases expressing hTERT developed cancer within 3-6 months, confirming the accuracy of molecular diagnosis. Another study has described positive molecular tests before cancer detection by conventional diagnostic systems (34). Moreover, Rehat et al reported telomerase activity in histopathologically-dysplastic tissues taken from TCC patients near the area of resected tumors (35). They interpreted the presence of telomerase activity as an indicator of the onset of the process of carcinogenesis in the tissue located at the periphery of the cancer field. In contrast to previous reports, none of the healthy volunteers in our study were found positive for hTERT expression $(21,32,36)$. Our findings are supported by the results of Melissourgos et al (31). The discrepancies between the different studies could be attributed to the existing variability in the sample selection. For example, Ito and colleagues had analyzed normal tissues lying adjacent to cancerous lesions, which occasionally carry the risk of incomplete tumor cell exclusion.

Recently, several assays have been developed for bladder cancer diagnosis using FISH technology, based on the detection of chromosomal aberrations present in exfoliated urothelial 
cells obtained from urine or bladder irrigation specimens. FISH has thus been regarded as a potential tool for the diagnosis of bladder cancer and the prediction of recurrence (20). In the current study, M-FISH increased the sensitivity rates obtained by urine cytology for bladder cancer detection across all histological grades and stages, with no significant difference in specificity. FISH shows its greatest potential in comparison to cytology when applied to superficial and low grade tumors, in which the appearance of the exfoliated tumor cells resembles normal urothelial cells, making cytology of little use in diagnosis (33.3 vs. $0 \%$ for pTa tumor stage and 50 vs. $16.7 \%$ for grade I tumors). Our results are supported by the studies of Halling et al and Placer et al $(11,20)$. On the other hand, M-FISH was markedly less sensitive than hTERT expression for the detection of superficial tumors at the pTa stage (33.3 vs. $66.7 \%)$. Halling et al reported that small urine sample volume or small-size tumors in which cells do not readily exfoliate may explain the low sensitivity of M-FISH in the case of pTa tumors (11). Moreover, previous studies have shown that more than $90 \%$ of low grade pTa tumors are diploid, with only a few showing chromosomal alterations (1).

In the present study, the homozygous deletion of $9 \mathrm{p} 21$ represented the most common chromosomal aberration in all cases collectively (63\% of cases). It was the only aberration detected in pTa tumors (33.3\% of cases), and the most common one in grade I cases $(66.6 \%$ deletion of $9 \mathrm{p} 21 \mathrm{vs.}$ $16.7 \%$ polysomy 3 ), suggesting that $9 \mathrm{p} 21$ deletion is one of the early events in bladder carcinogenesis. Similar results have been reported by Halling et al (11). On the other hand, polysomy 7 and polysomy 17 were mainly associated with advanced and high grade tumors. Similarly, previous studies have reported an association between polysomy 7 and 17 and highly aggressive tumor types $(6,37)$. One of the important target genes in polysomy 7 is monkey erythroblastosis oncogene-B (c-erbB)1, which codes for epidermal growth factor receptor (EGFR). Overexpression of EGFR has been strongly correlated with high stage and grade bladder cancer (38). Similarly, chromosome 17 harbors HER-2neu protooncogene, a gene which regulates the phosphorylation of B-catenin, leading to the inactivation of E-cadherin mediated cell adhesion and favoring invasive properties and metastatic potential (39).

In the present study, M-FISH was the most sensitive marker for the detection of tumor recurrence (100 vs. $40 \%$ for all other studied markers), suggesting that M-FISH could be used as a valuable predictor of tumor recurrence. Our results are supported by the study of Bubendorf et al, who reported that patients with M-FISH-negative urine are at a low risk of early recurrence and might be candidates for a reduced frequency of follow-up cytology (6).

In conclusion, the assessment of hTERT mRNA and chromosomal aberration (M-FISH) in exfoliated urothelium represents a highly sensitive tool for the early detection of bladder cancer. It seems conceivable that these emerging urine-based molecular assays could progressively replace urine cytology and form a non-invasive reliable adjunct to cyctoscopy for early bladder cancer diagnosis. The high sensitivity of FISH in the detection of recurrence suggests the use of FISH in combination with urine cytology as an alternative of cyctoscopy during follow-up.

\section{References}

1. El-Mawla NG, EL-Bolkainy MN and Khalid HM: Bladder cancer in Africa: update. Semin Oncol 28: 174-178, 2001.

2. Knowles MA: What we could do now: molecular pathology of bladder cancer. Br Med J 54: 215-221, 2001.

3. WHO Expert Committee on the Control of Schistosomiasis: Public health impact of schistosomiasis: disease and mortality. Bull World Health Organ 71: 657-662, 1993.

4. Muscheck M, Abol-Enein H, Chew K, Moor D, Bhargava V, Ghoneim MA, Carroll PR and Waldman FM: Comparison of genetic changes in Schistosome-related transitional and squamous bladder cancer using comparative genomic hybridization. Carcinogenesis 21: 1721-1726, 2000.

5. Shokeir AA: Squamous cell carcinoma of the bladder: pathology, diagnosis and treatment. BJU Int 93: 216-220, 2004.

6. Bubendorf L, Grilli B, Sauter G, Mihatsch MJ, Gasser TC and Dalquen P: Multiprobe FISH for enhanced detection of bladder cancer in voided urine specimens and bladder washings. Am J Clin Pathol 116: 79-86, 2001

7. Dey P: Urinary markers of bladder carcinoma. Clin Chim Acta 340: 57-65, 2004.

8. Dorkin TJ, Robson CN and Neal DE: The molecular pathology of urological malignancies. J Pathol 183: 380-387, 1997.

9. Jung I and Messing E: Molecular mechanisms and pathways in bladder cancer development and progression. Cancer Control 7: 325-334, 2000.

10. Jacques BK, Theo JMR, Goos NPM, et al: Real-time quantification of human telomerase reverse transcriptase mRNA in tumors and healthy tissues. Clin Chem 46: 313-318, 2000.

11. Halling KC, King W, Sokolova IA, Meyer RG, et al: A comparison of cytology and fluorescence in situ hybridization for the detection of urothelial carcinoma. J Urol 164: 1768-1775, 2000.

12. Eid MA, Kumar VM, Iczkowski KA, Bostwick DG and Tindall DJ: Expression of early growth response genes in human prostate cancer. Cancer Res 58: 2461-2468, 1998.

13. Gonzalez P, Zigler JS Jr, Epstein DL and Borrás T: Identification and isolation of differentially expressed genes from very small tissue samples. Biotechniques 26: 884-886, 1999.

14. Seville M, West AB, Cull MG and McHenry CS: Fluorometric assay for DNA polymerases and reverse transcriptase. Biotechniques 21: 664-672, 1996.

15. Kwok S and Higuchi R: Avoiding false positives with PCR. Nature 339: 237-238, 1989

16. Abdou SM, Jadayel DM, Min T, Swansbury GJ, Dainton MG, Jafer O, Powles RL and Catovsky D: Incidence of MLL rearrangement in acute myeloid leukemia, and a CALM-AF10 fusion in M4 type acute myeloblastic leukemia. Leuk Lymphoma 43: 89-95, 2002.

17. Dale JC, Masoner D, Fletcher T, Leaf-Brock S, Halling KC and Workman A: Fluorescence In Situ Hybridization for the Detection of Urothelial Carcinoma. Communiqué. A Mayo Reference Services Publication 27: 10, 2002.

18. Aviva P and Caroline S: Glossary of terms. In: Medical Statistics at a Glance. 2nd edition. Blackwell Publishing, pp144-152, 2005.

19. Marin-Aguilera M, Mengual L, Ribal MJ, Burset M, Arce Y, Ars E, Oliver A, Villavicencio H, Algaba F and Alcaraz A: Utility of a multiprobe fluorescence in situ hybridization assay in the detection of urothelial bladder cancer. Cancer Genet Cytogenet 173: 131-135, 2007

20. Placer J, Espinet B, Salido M, Sole F and Gelabert-Mas A: Clinical utility of a multiprobe FISH assay in voided urine specimens for the detection of bladder cancer and its recurrence, compared with urinary cytology. Eur Urol 42: 547-552, 2002.

21. Bialkowska-Hobrzanska H, Bowles L, Bukala B, et al: Comparison of human telomerase reverse transcriptase messenger RNA and telomerase activity as urine markers for diagnosis of bladder cancinoma. Mol Diagn 5: 267-277, 2000.

22. Van Rhijn BA, van der Poel HG and van der Kwast TH: Urine markers for bladder cancer surveillance: a systematic review. Eur Urol 47: 736-748, 2005.

23. Ghoneim MA: Bilharziasis of the genitourinary tract. BJU Int 89 (Suppl 1): 22-30, 2002.

24. Grossfeld GD and Carroll PR: Invasive bladder cancer. In: Comprehensive Urology. 1st edition. Weiss MR, George N Jr and O'Reilly PH (eds). Mosby International Ltd., London, Edinburgh, New York, Sydney and Toronto, pp373-393, 2001.

25. Inas Elattar: Bladder Cancer: Magnitude of the Problem. Annual Cancer Conference of the Egyptian Cancer Society, Danish Cancer Society and Aarhus University Hospital, Ain Sukhna, Egypt, 2005. 
26. Planz B, Jochims E, Deix T, Caspers HP, Jakse G and Boecking A: The role of urinary cytology for detection of bladder cancer. Eur $\mathrm{J}$ Surg Oncol 31: 304-308, 2005.

27. Khaled HM, Abdel-Salam I, Abdel-Gawad M, Metwally A, El-Demerdash S, El-Didi M, Morsi A and Ishak L: Evaluation of the BTA tests for the detection of Bilharzial related bladder cancer: the Cairo experience. Eur Urol 39: 91-94, 2001.

28. Serdar A: The importance of molecular tumor markers for bladder cancer. Turkish J Cancer 33: 171-176, 2003.

29. Lokeshwar VB and Soloway MS: Current bladder tumor tests: does their projected utility fulfill clinical necessity? J Urol 165: 1067-1077, 2001.

30. Oeda T and Manabe D: The usefulness of urinary FDP in the diagnosis of bladder cancer: comparison with NMP22, BTA and cytology. Nippon Hinyokika Gakkai Zasshi 92: 1-5, 2001.

31. Melissourgos N, Kastrinakis NG, Davilas I, Foukas A, Farmakis A and Lykourinas M: Detection of human telomerase reverse transcriptase mRNA in urine of patients with bladder cancer: evaluation of an emerging tumor marker. Urology 62: 362-367, 2003.

32. Neves M, Ciofu C, Larousserie F, et al: Prospective evaluation of genetic abnormalities and telomerase expression in exfoliated urinary cells for bladder cancer detection. J Urol 167: 1276-1281, 2002 .
33. Breslow RA, Shay JW, Gazdar AF and Srivastava S: Telomerase and early detection of cancer: a National Cancer Institute workshop. J Natl Cancer Inst 89: 618-623, 1997.

34. Wisman GBA, Hollema H, De Jong S, et al: Telomerase activity as a biomarker for pre-nuplastic cervical disease in scraping and frozen sections from patients with abnormal cervical smear. J Clin Oncol 16: 2238-2245, 1998.

35. Rehat MA, Lahar N, Gazawi H, et al: Telomerase activity in patients with transitional cell carcinoma. Cancer 85: 919-924, 1999.

36. Ito $\mathrm{H}$, Kyo $\mathrm{S}$, Kanaya $\mathrm{T}$, et al: Detection of human telomerase reverse transcriptase messenger RNA in voided urine samples as a useful diagnostic tool for bladder cancer. Clin Cancer Res 4: 2807-2810, 1998.

37. Sokolova IA, Halling KC, Jenkins RB, Burkhardt HM, Meyer RG, Seelig SA and King W: The development of a multitarget, multicolor fluorescence in situ hybridization assay for the detection of urothelial carcinoma in urine. J Mol Diagn 2: 116-123, 2000.

38. Bryan RT, Hussain SA, James ND, Jankowski JA, Wallace DM: Molecular pathways in bladder cancer: part 1. BJU Int 95: 485-490, 2005.

39. Hoff ER, Tubbs RR, Myles JL and Procop GW: HER2/neu amplification in breast cancer: stratification by tumor type and grade. Am Clin Pathol 117: 916-921, 2002. 